\title{
Spin polarizabilities and polarizabilities of the nucleon studied by free and quasi-free Compton scattering at MAMI (Mainz)*
}

\author{
Martin Schumacher \\ Zweites Physikalisches Institut der Universität, Tammannstraße 1 \\ D-37077 Göttingen, Germany \\ E-mail: schumacher@physik2.uni-goettingen.de
}

\begin{abstract}
In addition to the E2/M1 ratio of the $N \rightarrow \Delta$ transition, the electromagnetic polarizabilities and spin-polarizabilities are important structure constants of the nucleon which serve as sensitive tests of chiral perturbation theory and of models of the nucleon. Recently, these quantities have been investigated experimentally at MAMI (Mainz) by high-precision Compton scattering using hydrogen and deuterium targets, where for the latter the method of quasi-free scattering has been applied.
\end{abstract}

\section{Introduction}

Compton scattering is an excellent tool for studying the electromagnetic structure of the nucleon. This process supplements on information about photoexcitation of the internal degrees of freedom of the nucleon as contained in the amplitudes for meson photoproduction and supplies specific two-photon structure constants of the nucleon as there are the electric and magnetic polarizabilities $\alpha$ and $\beta$, respectively, and the spin-polarizabilities $\gamma_{0}$ and $\gamma_{\pi}$ for the forward and backward directions, respectively. These two-photon structure constants are special cases of the invariant (LPS) [1] amplitudes $A_{i}(\nu, t)(i=1 \cdots 6)$ which, therefore, may be considered as generalized polarizabilities. One motivation for investigating the generalized polarizabilities $A_{i}$ is that insight is obtained into the physical nature of the two-photon structure constants. Specifically, t-channel phenomena may be investigated as there are the $\sigma$ meson contribution to $\alpha-\beta$ and the $\pi^{0}, \eta$ and $\eta^{\prime}$ meson contributions to $\gamma_{\pi}$. While the t-channel part of $\gamma_{\pi}$ appears to be well understood, the corresponding part of $\alpha-\beta$ is still under investigation and especially awaits consideration in chiral perturbation theory. Recently, deuteron targets have been used in measurements of quasi-free Compton scattering by the nucleons [2, 3] bound in the deuteron, thus leading to the first precise results for the polarizabilities of the neutron. In contrast to the competing methods of electromagnetic scattering of neutrons in a Coulomb field and coherent elastic (Compton) scattering of photons by the deuteron where model uncertainties cannot be excluded, the method of quasi-free scattering has been tested and found valid through free and quasi-free Compton scattering experiments on the proton.

\section{Polarizabilities and invariant amplitudes}

In the extreme forward $(\theta=0)$ and extreme backward directions $(\theta=\pi)$ the amplitudes for Compton scattering may be written in the form [1, 4]

$$
\begin{aligned}
& T^{\mathrm{LAB}}(\theta=0)=f_{0}(\omega) \epsilon^{\prime} \cdot \epsilon+g_{0}(\omega) \mathrm{i} \sigma \cdot\left(\epsilon^{\prime} \times \epsilon\right) \\
& T^{\mathrm{LAB}}(\theta=\pi)=f_{\pi}(\omega) \epsilon^{\prime} \cdot \epsilon+g_{\pi}(\omega) \mathrm{i} \sigma \cdot\left(\epsilon^{\prime} \times \epsilon\right) .
\end{aligned}
$$

where $f(\omega)=1 / 2\left\{T_{1 / 2}+T_{3 / 2}\right\}$ correspond to the case where the initial-state and final-state photons have parallel linear polarization and $g(\omega)=1 / 2\left\{T_{1 / 2}-T_{3 / 2}\right\}$ to the case where these photons have perpendicular linear polarization. The relations between the amplitudes

${ }^{*}$ Proceedings of GDH 2002, Genova, Italy 3-6 July 2002, M. Anghinolfi, M. Battaglieri, R. De Vita, Eds., World Scientific 2003 (updated version)

Supported by Deutsche Forschungsgemeinschaft 
$f$ and $g$ and the invariant (LPS) [1] amplitudes $A_{i}$ are [4]

$$
\begin{aligned}
& f_{0}(\omega)=-\frac{\omega^{2}}{2 \pi}\left[A_{3}(\nu, t)+A_{6}(\nu, t)\right], \\
& g_{0}(\omega)=\frac{\omega^{3}}{2 \pi m} A_{4}(\nu, t), \\
& f_{\pi}(\omega)=-\frac{\omega \omega^{\prime}}{2 \pi}\left(1+\frac{\omega \omega^{\prime}}{m^{2}}\right)^{1 / 2}\left[A_{1}(\nu, t)+\frac{\omega \omega^{\prime}}{m^{2}} A_{5}(\nu, t)\right], \\
& g_{\pi}(\omega)=-\frac{\omega \omega^{\prime}}{2 \pi}\left(1+\frac{\omega \omega^{\prime}}{m^{2}}\right)^{-1 / 2} \frac{\omega+\omega^{\prime}}{2 m}\left[A_{2}(\nu, t)+\left(1+\frac{\omega \omega^{\prime}}{m^{2}}\right) A_{5}(\nu, t)\right], \\
& \omega^{\prime}(\theta=\pi)=\frac{\omega}{1+2 \frac{\omega}{m}}, \nu=\frac{1}{2}\left(\omega+\omega^{\prime}\right), t(\theta=0)=0, t(\theta=\pi)=-4 \omega \omega .
\end{aligned}
$$

For the polarizabilities we obtain the relations

$$
\begin{aligned}
& \alpha+\beta=-\frac{1}{2 \pi}\left[A_{3}^{\mathrm{nB}}(0,0)+A_{6}^{\mathrm{nB}}(0,0)\right], \alpha-\beta=-\frac{1}{2 \pi}\left[A_{1}^{\mathrm{nB}}(0,0)\right], \\
& \gamma_{0}=\frac{1}{2 \pi m}\left[A_{4}^{\mathrm{nB}}(0,0)\right], \gamma_{\pi}=-\frac{1}{2 \pi m}\left[A_{2}^{\mathrm{nB}}(0,0)+A_{5}^{\mathrm{nB}}(0,0)\right],
\end{aligned}
$$

where $A_{i}^{\mathrm{nB}}$ are the non-Born parts of the amplitudes $A_{i}$.

Measurements of spin independent and spin dependent total photoabsorption cross sections have been carried out. Using the sum rules

$$
\alpha+\beta=\frac{1}{2 \pi^{2}} \int_{\omega_{t h r}}^{\infty} \sigma_{t o t}(\omega) \frac{d \omega}{\omega^{2}} \quad \text { and } \quad \gamma_{0}=\frac{1}{4 \pi^{2}} \int_{\omega_{t h r}}^{\infty} \frac{\sigma_{1 / 2}(\omega)-\sigma_{3 / 2}(\omega)}{\omega^{3}} d \omega,
$$

respectively, the following results have been obtained [5, 6, 7]

$$
\alpha_{p}+\beta_{p}=14.0 \pm 0.3, \quad \alpha_{n}+\beta_{n}=15.2 \pm 0.5, \quad \gamma_{0}^{(p)}=-0.86 \pm 0.13
$$

in units of $10^{-4} \mathrm{fm}^{3}$ for polarizabilities and $10^{-4} \mathrm{fm}^{4}$ for the spin polarizabilities. The number given for the spin polarizability $\gamma_{0}^{(p)}$ contains sizable corrections for low and high energy contributions not covered by the experiment, the errors of which have not been taken into account.

\section{$3 \quad$ Asymptotic amplitudes}

According to the Landau-Yang theorem 8 particles with $J^{P C}=0^{++}, 2^{++}, 4^{++}, \cdots$ decay into two photons with parallel directions of linear polarization and particles with $J^{P C}=$ $0^{-+}, 2^{-+}, 4^{-+}, \cdots$ into two photons with perpendicular directions of linear polarization for the case of total helicity $\lambda=\lambda_{\gamma_{2}}-\lambda_{\gamma_{1}}=0$. This leads to the conclusion that mainly the $\sigma(600)$ meson may be a $t$-channel contribution to $f_{\pi}(\omega)$, whereas mainly the $\pi(135), \eta(547)$ and $\eta^{\prime}(958)$ mesons may be $t$-channel contributions to $g_{\pi}(\omega)$. For the case of total helicity $\lambda=\lambda_{\gamma_{2}}-\lambda_{\gamma_{1}}=2$ the corresponding quantum numbers are $J^{P C}=2^{++}, 4^{++}, 6^{++}, \cdots$ and $J^{P C}=3^{++}, 5^{++}, 7^{++}, \cdots$. From this it may be concluded that the Pomeron $\mathcal{P}$, the $f_{2}(1270)$ and the $a_{2}(1320)$ are $t$-channel contributions to $f_{0}(\omega)$ which are used to parametrize the total photoabsorption cross section based on a Regge ansatz and, thus, are already taken care of by the $s$-channel. A natural extension of these considerations is that $J^{P C}=3^{++}$mesonic or gluonic intermediate states may be $t$-channel contributions to the Gerasimov-Drell-Hearn 9] amplitude $g_{0}(\omega)$.

\section{Results of Compton scattering by the proton and the neutron}

The four polarizabilities (3) are defined for the forward $(\theta=0)$ and backward $(\theta=\pi)$ directions and for low energies. Experiments, however, require intermediate angles and 
energies where the terms of higher order in $\omega$ are not negligible. Therefore, the Compton scattering process has to be investigated in general. In the second resonance region and at backward angles the data mainly serve as a test of the $\sigma$-pole ansatz [1] for the asymptotic part of $f_{\pi}$ and for the determination of the relevant mass-parameter $m_{\sigma}$ of this ansatz. A good fit to the data of the second resonance region is obtained [10, 11] if a parameter of $m_{\sigma}=600 \mathrm{MeV}$ is applied. This result appears to be quite satisfactory because this number is in agreement with results of other investigations. Nevertheless, more precise data extending to higher energies and disentangling the amplitudes $f_{\pi}$ and $g_{\pi}$ via polarized photons would be desirable. This makes Compton scattering in the second resonance region an ideal project for the polarized photon beams at the $1.5 \mathrm{GeV}$ accelerator MAMI C (Mainz) and at GRAAL (Grenoble).

Compton scattering by the proton at energies below $\pi$ threshold using the large-angle arrangement TAPS led to a determination of $\alpha_{p}-\beta_{p}$ with unprecedented precision [12. The result obtained

$$
\left.\alpha_{p}-\beta_{p}=10.5 \pm 0.9(\text { stat }+ \text { syst }) \pm 0.7 \text { (model }\right)
$$

serves as a standard input for all analyses of Compton scattering by the proton in terms of dispersion theories.

Precise results for the electromagnetic polarizabilities of the neutron have been obtained for the first time in a recent experiment on quasi-free Compton scattering by the neutron carried out 2] using the large Mainz $48 \mathrm{~cm} \oslash \times 64 \mathrm{~cm} \mathrm{NaI(Tl)}$ detector and the Göttingen segmented neutron detector SENECA in coincidence. Furthermore, quasi-free Compton scattering by the proton and Compton scattering by the free proton have been analyzed as tests of the method. As a byproduct the experiment carried out for the free proton served to determine the spin polarizability $\gamma_{\pi}^{(p)}$ leading to 13

$$
\gamma_{\pi}^{(p)}=(-38.7 \pm 1.8)
$$

The essential conclusion drawn from this result is that the spin polarizability $\gamma_{\pi}^{(p)}$ is in agreement with the prediction of dispersion theory (3) and not in strong disagreement as observed in [14. Therefore, for the analysis of the quasi-free double differential cross sections for the neutron shown in the left panel of Fig. 1 we also may assume, that $\gamma_{\pi}^{(n)}$ is in agreement with the prediction of dispersion theory, leading to the value given in the figure caption. The fit to the double differential cross sections then led to the result

$$
\alpha_{n}-\beta_{n}=9.8 \pm 3.6(\text { stat })_{-1.1}^{+2.1}(\text { syst }) \pm 2.2(\text { model }) .
$$

It is of interest to note that the number adopted for $\gamma_{\pi}^{(n)}$ is confirmed by the experiment as is shown in the right panel of Fig. 1 where the two parameter $\alpha_{n}-\beta_{n}$ and $\gamma_{\pi}^{(n)}$ are varied independently. Making use of the $\chi^{2}$ distribution we arrive at

$$
\gamma_{\pi}^{(n)}=58.6 \pm 4.0
$$

A summary of the status of electromagnetic polarizabilities is given in the followig table. The experimental data for $\alpha, \beta$ and $\gamma_{\pi}$ are due to recent experimental work carried out at MAMI using LARA [10, 11], TAPS [12] and the large Mainz NAI(Tl) combined with the SENECA detector [2] 13]. The value for $\gamma_{\pi}(t-$ channel $)$ is based on the prediction provided by (10), the two values for $\gamma_{\pi}(s-$ channel $)$ labeled "present work" and "sum rule" on (11) and (12), respectively:

$$
\begin{aligned}
& \gamma_{\pi}(t-\text { channel })=\frac{1}{2 \pi m}\left[\frac{g_{\pi N N} F_{\pi^{0} \gamma \gamma}}{m_{\pi^{0}}^{2}} \tau_{3}+\frac{g_{\eta N N} F_{\eta \gamma \gamma}}{m_{\eta}^{2}}+\frac{g_{\eta^{\prime} N N} F_{\eta^{\prime} \gamma \gamma}}{m_{\eta^{\prime}}^{2}}\right], \\
& \gamma_{\pi}(s-\text { channel })=-\frac{1}{2 \pi m}\left[A_{2}^{\text {int }}(0,0)+A_{5}^{\text {int }}(0,0)\right], \\
& \gamma_{\pi}(s-\text { channel })=\int_{\omega_{0}}^{\infty} \sqrt{1+\frac{2 \omega}{m}}\left(1+\frac{\omega}{m}\right) \sum_{n} P_{n}\left[\sigma_{3 / 2}^{n}(\omega)-\sigma_{1 / 2}^{n}(\omega)\right] \frac{d \omega}{4 \pi^{2} \omega^{3}},
\end{aligned}
$$



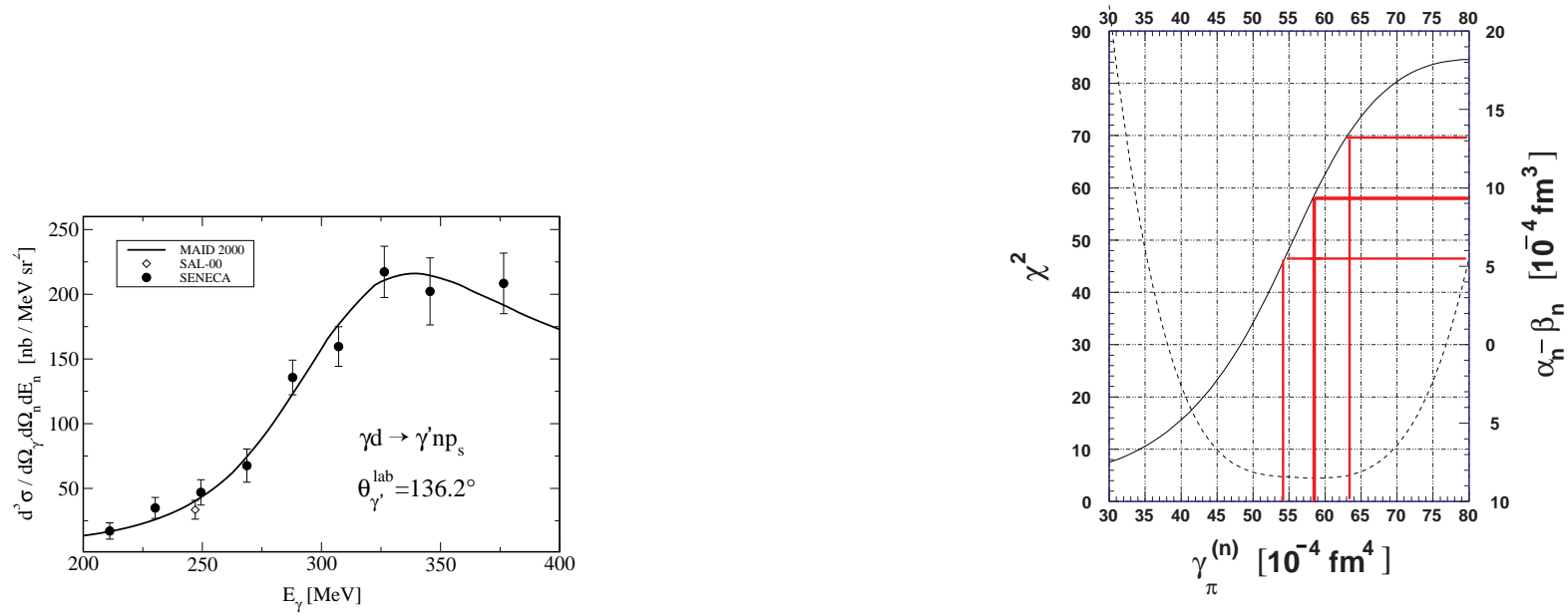

Figure 1: left: Triple differential cross section for Compton scattering by the neutron compared with prediction using $\alpha_{n}-\beta_{n}=9.8 \times 10^{-4} \mathrm{fm}^{3}$ and $\gamma_{\pi}^{(n)}=58.6 \times 10^{-4} \mathrm{fm}^{4}$. right: $\chi^{2}$ and $\alpha_{n}-\beta_{n}$ $\operatorname{versus} \gamma_{\pi}^{(n)}$

Table 1: Summary of polarizabilities of proton and neutron

\begin{tabular}{llll}
\hline & proton & neutron & \\
\hline$\alpha$ & $12.2 \pm 0.6$ & $12.5 \pm 2.3$ & experiment \\
$\beta$ & $1.8 \mp 0.6$ & $2.7 \mp 2.3$ & experiment \\
$\gamma_{\pi}$ & $-38.7 \pm 1.8$ & $+58.6 \pm 4.0$ & experiment \\
$\gamma_{\pi}$ & $-39.5 \pm 2.4$ & $+52.5 \pm 2.4$ & sum rule [15] \\
\hline$\gamma_{\pi}(\mathrm{t}-$ channel $)$ & -46.6 & +43.4 & $\pi^{0}+\eta+\eta^{\prime}$ \\
$\gamma_{\pi}(\mathrm{s}-$ channel $)$ & $+7.9 \pm 1.8$ & $+15.2 \pm 4.0$ & present work \\
$\gamma_{\pi}(\mathrm{s}-$ channel $)$ & $+7.1 \pm 1.8$ & $+9.1 \pm 1.8$ & sum rule [15] \\
\hline
\end{tabular}

where $A_{i}^{\text {int }}$ denotes the integral part of the amplitude and $P_{n}= \pm 1$ the relative parity of the final state $n$ with respect to the target [15].

There is good agreement between the present work and the sum rule prediction [15] for $\gamma_{\pi}(s-$ channel $)$ in case of the proton but some discrepancy between the corresponding numbers obtained for the neutron. We believe that this discrepancy is caused by internal inconsistencies of the photomeson amplitudes available for the neutron.

\section{References}

[1] A.I. L'vov, V.A. Petrun'kin, M. Schumacher, Phys. Rev. C 55, 359 (1997)

[2] K. Kossert et al., Phys. Rev. Lett. 88, 162301 (2002); Eur. Phys. J. A 16, 259 (2003)

[3] M.I. Levchuk, A.I. L'vov, V.A. Petrun'kin, Few-Body Syst. 16, 101 (1994)

[4] B. Babusci, G. Giordano, A.I. L'vov, G. Matone, A.M. Nathan, Phys. Rev. C 58, 1013 (1998)

[5] M.I. Levchuk, A.I. L'vov, Nucl. Phys. A 674, 449 (2000)

[6] J. Ahrens et al., Phys. Rev. Lett. 84, 5950 (2000)

[7] J. Ahrens et al., Phys. Rev. Lett. 87, 022003 (2001)

[8] C.N. Yang, Phys. Rev. 77 (1950) 242; A.I. L'vov, private communication (2003)

[9] S.B. Gerasimov, Sov. J. Nucl. Phys. 2, 430 (1966)

S.D. Drell and A.C. Hearn, Phys. Rev. Lett. 16, 908 (1966) 
[10] G. Galler et al., Phys. Lett. B 503, 245 (2001)

[11] S. Wolf et al., Eur. Phys. J. A 12, 231 (2001)

[12] V. Olmos de León et al., Eur. Phys. J. A 10, 207 (2001)

[13] M. Camen et al., Phys. Rev. C 65, 032202(R) (2002)

[14] J. Tonnison et al., Phys. Rev. Lett. 80, 4382 (1998)

G. Blanpied et al., Phys. Rev. C 64, 025203 (2001)

[15] A.I. L'vov, A.M. Nathan, Phys. Rev. C 59, 1064 (1999) 